\title{
Liouville type theorem for a singular elliptic equation with finite Morse index
}

\author{
Zonghu Xiu ${ }^{1 *}$, Jing Zhao ${ }^{1}$, Jianyi Chen ${ }^{1}$ and Hongwei Yang ${ }^{2}$
}

\footnotetext{
"Correspondence: qingda@163.com 1 Science and Information College, Qingdao Agricultural University, Qingdao, P.R. China

Full list of author information is available at the end of the article
}

\section{Abstract \\ This paper considers the nonexistence of solutions for the following singular quasilinear elliptic problem:$$
\left\{\begin{array}{l}
-\operatorname{div}\left(|x|^{-a p}|\nabla u|^{p-2} \nabla u\right)=f(|x|)|u|^{r-1} u, \quad x \in \mathbb{R}_{+\prime}^{N} \\
|x|^{-a p}|\nabla u|^{p-2} \frac{\partial u}{\partial v}=g(|x|)|u|^{q-1} u, \quad \text { on } \partial \mathbb{R}_{+^{\prime}}^{N}
\end{array}\right.
$$ \\ where $\mathbb{R}_{+}^{N}=\left\{x=\left(x^{\prime}, x_{N}\right) \mid x^{\prime} \in \mathbb{R}^{N-1}, x_{N}>0\right\}$ and $\partial \mathbb{R}_{+}^{N}=\left\{x=\left(x^{\prime}, x_{N}\right) \mid x^{\prime} \in \mathbb{R}^{N-1}, x_{N}=0\right\}$. When the weight functions satisfy some suitable assumptions, we prove that problem (0.1) has no nontrivial bounded solutions with finite Morse index.}

Keywords: Liouville theorem; Morse index; Singular elliptic equation

\section{Introduction and main results}

In this paper, we consider the following problem:

$$
\left\{\begin{array}{l}
-\operatorname{div}\left(|x|^{-a p}|\nabla u|^{p-2} \nabla u\right)=f(|x|)|u|^{r-1} u, \quad x \in \mathbb{R}_{+}^{N}, \\
|x|^{-a p}|\nabla u|^{p-2} \frac{\partial u}{\partial v}=g(|x|)|u|^{q-1} u, \quad \text { on } \partial \mathbb{R}_{+}^{N},
\end{array}\right.
$$

where $a>0, r>1, q>1, p \geq 2$ and $\mathbb{R}_{+}^{N}=\left\{x=\left(x^{\prime}, x_{N}\right) \mid x^{\prime} \in \mathbb{R}^{N-1}, x_{N}>0\right\}$ denotes the upper half-space in $\mathbb{R}^{N}$.

Liouville type theorems have been widely applied to research the nonexistence of nontrivial solutions for elliptic equations. Liouville theorem was first announced in 1844 by Liouville [1] for the special case of a doubly periodic function. The classical Liouville-type theorem states that a bounded harmonic (or holomorphic) function defined in the entire space $\mathbb{R}^{N}$ must be constant. Liouville type theorems for solutions with finite Morse indices have been widely studied in the past few decades. The idea of using Morse index of a solution to study a semilinear elliptic equation was first explored by Bahri and Lions in [2], where the following problem was considered on the half-space:

$$
\left\{\begin{array}{l}
-\Delta u=|u|^{p-1} u, \quad x \in \mathbb{R}_{+}^{N}, \\
u=0, \quad \text { on } \partial \mathbb{R}_{+}^{N} .
\end{array}\right.
$$

(c) The Author(s) 2019. This article is distributed under the terms of the Creative Commons Attribution 4.0 International License (http://creativecommons.org/licenses/by/4.0/), which permits unrestricted use, distribution, and reproduction in any medium, provided you give appropriate credit to the original author(s) and the source, provide a link to the Creative Commons license, and indicate if changes were made. 
The authors proved that (1.2) has no nontrivial bounded solution with finite Morse index when $1<p<\frac{N+2}{N-2}$. Later, many authors considered the positive solutions of (1.2) by some delicate methods. In [3], Chen and Li considered the positive solutions of (1.2) by the moving plane method. The authors first proved that the solution is symmetric and constant, then deduced that this constant is just zero. Inspired by the idea in [3], many scholars applied similar methods to research solutions of elliptic equations, see [4-7] and the references therein. $\mathrm{Yu}$ [8] studied (1.2) with a Neumann boundary condition. By using an energy estimate and Pohozaev identity, the author gave a result on the nonexistence of a finite Morse index solution.

In [9], Gidas and Spruck considered the elliptic problem

$$
-\Delta u=|x|^{a}|u|^{p-1} u \quad \text { in } \Omega .
$$

If $a=0$, the authors proved that (1.3) has no positive solutions if and only if $1<p<\frac{N+2}{N-2}$ $(=\infty$ if $N=2)$. If $a \neq 0$, problem (1.3) is complicated and less is known. For $a \leq-2$, the authors in [9] established an important result that (1.3) does not possess positive solutions in any domain $\Omega$ containing the origin. For $a>-2$, however, problem (1.3) is difficult and there are fewer results since some classical techniques fail for this case. In [10], Phan and Souple studied the positive bounded solution of (1.3) for the special case $a>0$ and $N=3$. The authors proved that (1.3) has no positive bounded solution in $\Omega=\mathbb{R}^{N}$ for $1<p<p_{s}(a)=(N+2+2 a) /(N-2)(=\infty$ if $N=2)$. In [11], Dancer et al. also studied problem (1.3) with $a>-2$, and classified the existence and behavior at infinity of positive solutions with a finite Morse index. In order to get the results on finite Morse index solutions, a duality method was applied in [11]. It is worth noting that the result on radial solutions of problem (1.3) is complete, see the following proposition in $[9,12]$.

Proposition A Let $N \geq 2, a>-2$ and $p>1$.

(i) If $p<p_{s}(a)$, then (1.3) has no positive radial solution in $\Omega=\mathbb{R}^{N}$.

(ii) If $p \geq p_{s}(a)$, then (1.3) possesses a bounded, positive radial solution in $\Omega=\mathbb{R}^{N}$.

For other manuscripts on Liouville-type theorems for nonlinear elliptic equations, we refer the readers to [13-20].

In recent years, a Liouville-type theorem for a higher order equation was also studied. $\mathrm{Hu}[21]$ considered the fourth order elliptic equation

$$
-\Delta^{2} u=|x|^{a}|u|^{p-1} u \text { in } \Omega .
$$

Applying the monotonicity formula and blowing down sequence, the author established a Liouville-type theorem for finite Morse index solutions. In [22], Dávila et al. studied (1.4) for the case $a=0$ and $\Omega=\mathbb{R}^{N}$. The authors gave a complete classification of finite Morse index solutions. Theorem 1.3 in [22] generalized a similar result of Farina in [23] for the classical Lane-Emden equation.

Some scholars applied the Liouville-type theorem for elliptical equations with the $p$ Laplace operator. In [24], the authors considered the following $p$-Laplace elliptic equations with exponential growth

$$
-\Delta_{p} u=f(x) e^{u}, \quad x \in \mathbb{R}^{N} .
$$


There are few works on the elliptic equation with the $p$-Laplace operator and exponential growth. By choosing a special test function, the authors gave the result on the nonexistence of positive stable solution for (1.5).

In our paper, we consider solutions of (1.1) in Sobolev space $W^{1, p}\left(|x|^{-a p}, \mathbb{R}_{+}^{N}\right)$. The weight functions $f(|x|)$ and $g(|x|)$ in (1.1) are radial. We are interested in the nonexistence of solutions with a finite Morse index. Our proofs in this paper are partly motivated by [14]. Since $a>0$, problem (1.1) is singular at $x=0$, and we need more a delicate energy estimate and computations. We want to point out that the solutions in our problem (1.1) may change sign, thus the moving plane method mentioned above does not work.

Denote by $J(u)$ the natural functional to problem (1.1), that is,

$$
\begin{aligned}
J(u)= & \frac{1}{p} \int_{\mathbb{R}_{+}^{N}}|x|^{-a p}|\nabla u|^{p} d x-\frac{1}{r+1} \int_{\mathbb{R}_{+}^{N}} f(|x|)|u|^{r+1} d x \\
& -\frac{1}{q+1} \int_{\partial \mathbb{R}_{+}^{N}} g\left(\left|x^{\prime}\right|\right)|u|^{q+1} d x^{\prime} .
\end{aligned}
$$

We define the function

$$
Q_{u}(\varphi)=\int_{\mathbb{R}_{+}^{N}}|x|^{-a p}|\nabla u|^{p} d x+(p-2) \int_{\mathbb{R}_{+}^{N}}|x|^{-a p}|\nabla u|^{p-4}(\nabla u \cdot \nabla \varphi)^{2} d x .
$$

It is well known that the Morse index $i(u)$ is defined as the maximal dimension of all subspaces $X \in C_{0}^{1}\left(\mathbb{R}^{N}\right)$ such that $Q_{u}(\varphi)<0$.

In order to get our result, we make the following assumptions:

$\left(A_{1}\right)$ There exist $b_{0}>0, d_{0}>0, b>\frac{N(2-p)-2 p(a+1)}{p}$ and $d>\frac{(2-p) N}{p}-2 a-1$ such that $f(|x|)|x|^{-b} \rightarrow b_{0}$ and $g(|x|)|x|^{-d} \rightarrow d_{0}$ as $|x| \rightarrow \infty$.

$\left(A_{2}\right) f(|x|) \in C^{1}\left(\mathbb{R}_{+}^{N} \backslash\{0\}\right)$ is radial and nonnegative in $\mathbb{R}_{+}^{N}$, and $g(|x|) \in C^{1}\left(\partial \mathbb{R}_{+}^{N} \backslash\{0\}\right)$ is radial and nonnegative in $\partial \mathbb{R}_{+}^{N}$.

$\left(A_{3}\right)$ The functions $f(\tau)$ and $g(\tau)$ satisfy

$$
\left(\tau^{\mu} f(\tau)\right)^{\prime}>0, \quad \forall \tau=|x| \in \mathbb{R}_{+}^{N} \backslash\{0\}, \quad\left(\tau^{\omega} g(\tau)\right)^{\prime}>0, \quad \forall \tau=|x| \in \partial \mathbb{R}_{+}^{N} \backslash\{0\},
$$

where $\mu=[N p-(r+1)(1+a-N)] / p$ and $\omega=[(N-1) p-(q+1)(N-p-p a)] / p$.

Our main result on (1.1) is listed below.

Theorem 1 Assume $N \geq 2$ and suppose functions $f(\tau)$ and $g(\tau)$ satisfy assumptions $\left(A_{1}\right)$ $\left(A_{3}\right)$. Let $u \in W^{1, p}\left(|x|^{-a p}, \mathbb{R}_{+}^{N}\right)$ be a bounded solution of $(1.1)$. If $i(u)<\infty$, then $u \equiv 0$ in $\mathbb{R}_{+}^{N}$.

This paper is organized as follows. In Sect. 2, we establish several lemmas and estimates. In Sect. 3, we give a Pohozaev identity and then complete the proof of Theorem 1.

\section{Preliminary results}

In order to study the solutions with a finite Morse index, we will establish several lemmas. We first define a cut-off function $\varphi_{\tau, s} \in[0,1]$ as

$$
\varphi_{\tau, s}(|x|)= \begin{cases}0, & |x|<\tau \text { or }|x|>2 s \\ 1, & 2 \tau \leq|x| \leq s\end{cases}
$$

Furthermore, $\left|\nabla \varphi_{\tau, s}(|x|)\right| \leq \frac{2}{\tau}$ for $\tau<|x| \leq 2 \tau$ and $\left|\nabla \varphi_{\tau, s}(|x|)\right| \leq \frac{2}{s}$ for $s<|x|<2 s$. 
Lemma 2.1 Assume $u(x)$ is a solution of (1.1) with a finite Morse index, then there exists $\tau_{0}>0$ such that $Q_{u}\left(u \varphi_{\tau_{0}, s}(|x|)\right) \geq 0$.

Proof Let $i(u)=k$ and $g(\tau, s)=Q_{u}\left(u \varphi_{\tau, s}(|x|)\right)$, where $s>2 \tau>2 \tau_{0}>0$. Assume on the contrary that there exist $s_{m} \rightarrow \infty, \tau_{m} \rightarrow \infty$ such that $s_{m+1}>2 \tau_{m+1}>\tau_{m+1}>2 s_{m}$ and

$$
g\left(r_{m}, s_{m}\right)=Q_{u}\left(u \varphi_{\tau_{m}, s_{m}}\right)<0 \quad \text { for } m=1,2, \ldots
$$

Then, one gets from (2.2) that $u \varphi_{\tau_{m}, s_{m}} \not \equiv 0$ for $\forall 1 \leq m \leq k+1$. Note that $\left\{u \varphi_{\tau_{m}, s_{m}}\right\}_{m=1}^{k+1}$ have disjoint support, which implies that $\left\{u \varphi_{\tau_{m}, s_{m}}\right\}_{m=1}^{k+1}$ are orthogonal in $L^{2}\left(\mathbb{R}^{N}\right)$ and linearly independent, so the dimension of the space

$$
M_{k+1}=\operatorname{span}\left\{u \varphi_{\tau_{m}, s_{m}}\right\}_{m=1}^{k+1}
$$

is $k+1$. Furthermore, one gets from (2.2) that $Q_{u}(h)<0$ for any $h \in M_{k+1}$. Thus, the Morse index of $u$ is at least $k+1$, which contradicts $i(u)=k$, and we complete the proof of Lemma 2.1.

Now, we give some estimates.

Lemma 2.2 Assume $\left(A_{1}\right)-\left(A_{3}\right)$. If $u$ is a bounded solution of (1.1) with a finite Morse index, then

$$
\int_{\mathbb{R}_{+}^{N}} f(|x|)|u|^{r+1} d x<\infty, \quad \int_{\partial \mathbb{R}_{+}^{N}} g\left(\left|x^{\prime}\right|\right)|u|^{q+1} d x^{\prime}<\infty .
$$

Proof We prove the first claim of (2.4). For this purpose, we will divide our proof into three cases.

(i) $r>p-1+\frac{b p}{N}$.

According to Lemma 2.1, there exists $\tau_{0}>0$ such that $Q_{u}\left(u \varphi_{\tau_{0}, s}\right) \geq 0$ for $s>2 \tau_{0}$, that is,

$$
\begin{aligned}
& q \int_{\partial \mathbb{R}_{+}^{N}} g\left(\left|x^{\prime}\right|\right)|u|^{q+1} \varphi_{\tau_{0}, s} d x^{\prime}+r \int_{\mathbb{R}_{+}^{N}} f(|x|) \varphi_{\tau_{0}, s}^{2} d x \\
& \quad \leq \int_{\mathbb{R}_{+}^{N}}|x|^{-a p}|\nabla u|^{p-2}\left(\nabla u \varphi_{\tau_{0}, s}+u \nabla \varphi_{\tau_{0}, s}\right)^{2} d x \\
& \quad+(p-2) \int_{\mathbb{R}_{+}^{N}}|x|^{-a p}|\nabla u|^{p-4}\left(\nabla u \cdot \nabla\left(u \varphi_{\tau_{0}, s}\right)\right)^{2} d x \\
& \quad=(p-1) \int_{\mathbb{R}_{+}^{N}}|x|^{-a p}|\nabla u|^{p} \varphi_{\tau_{0}, s}^{2} d x+2(p-1) \int_{\mathbb{R}_{+}^{N}}|x|^{-a p}|\nabla u|^{p-2} u \varphi_{\tau_{0}, s} \nabla u \nabla \varphi_{\tau_{0}, s} d x \\
& \quad+(p-1) \int_{\mathbb{R}_{+}^{N}}|\nabla u|^{2}|\nabla \varphi|_{\tau_{0}, s}^{2} d x .
\end{aligned}
$$

On the other hand, multiplying (1.1) by $u \varphi_{\tau_{0}, s}^{2}$ and integrating by parts, one gets

$$
\begin{aligned}
& \int_{\mathbb{R}_{+}^{N}} f(|x|)|u|^{r+1} \varphi_{\tau_{0}, s}^{2} d x+\int_{\partial \mathbb{R}_{+}^{N}} g\left(\left|x^{\prime}\right|\right)|u|^{q+1} \varphi_{\tau_{0}, s}^{2} d x^{\prime} \\
& =\int_{\mathbb{R}_{+}^{N}}|x|^{-a p}|\nabla u|^{p} \varphi_{\tau_{0}, s}^{2} d x \\
& \quad+2 \int_{\mathbb{R}_{+}^{N}}|x|^{-a p}|\nabla u|^{p-2} u \varphi_{\tau_{0}, s} \nabla u \nabla \varphi_{\tau_{0}, s} d x .
\end{aligned}
$$


It follows from Lemma 2.1 that there exists $\tau_{0}>0$ such that

$$
\begin{aligned}
& q \int_{\partial \mathbb{R}_{+}^{N}} g\left(\left|x^{\prime}\right|\right)|u|^{q+1} \varphi_{\tau_{0}, s} d x^{\prime}+r \int_{\mathbb{R}_{+}^{N}} f(|x|)|u|^{r+1} \varphi_{\tau_{0}, s}^{2} d x \\
& \leq \quad(p-1) \int_{\mathbb{R}_{+}^{N}}|x|^{-a p}|\nabla u|^{p} \varphi_{\tau_{0}, s}^{2} d x \\
& \quad+2(p-1) \int_{\mathbb{R}_{+}^{N}}|x|^{-a p}|\nabla u|^{p-2} u \varphi_{\tau_{0}, s} \nabla u \nabla \varphi_{\tau_{0}, s} d x \\
& \quad+(p-1) \int_{\mathbb{R}_{+}^{N}}|x|^{-a p}|\nabla u|^{p-2} u^{2}\left|\nabla \varphi_{\tau_{0}, s}\right|^{2} d x .
\end{aligned}
$$

Inserting (2.6) into (2.7), one gets

$$
\begin{aligned}
& q \int_{\partial \mathbb{R}_{+}^{N}} g\left(\left|x^{\prime}\right|\right)|u|^{q+1} \varphi_{\tau_{0}, s} d x^{\prime}+r \int_{\mathbb{R}_{+}^{N}} f(|x|)|u|^{r+1} \varphi_{\tau_{0}, s}^{2} d x \\
& \leq(p-1)\left[\int_{\mathbb{R}_{+}^{N}} f(|x|)|u|^{r+1} \varphi_{\tau_{0}, s}^{2} d x\right. \\
& \left.\quad+\int_{\partial \mathbb{R}_{+}^{N}} g(|x|)|u|^{q+1} \varphi_{\tau_{0}, s}^{2} d x^{\prime}\right]+(p-1) \int_{\mathbb{R}_{+}^{N}}|x|^{-a p}|\nabla u|^{p-2} u^{2}\left|\nabla \varphi_{\tau_{0}, s}\right|^{2} d x .
\end{aligned}
$$

That is,

$$
\begin{aligned}
& (q-p-1) \int_{\partial \mathbb{R}_{+}^{N}} g\left(\left|x^{\prime}\right|\right)|u|^{q+1} \varphi_{\tau_{0}, s} d x^{\prime}+(r-p-1) \int_{\mathbb{R}_{+}^{N}} f(|x|)|u|^{r+1} \varphi_{\tau_{0}, s}^{2} d x \\
& \leq(p-1) \int_{\mathbb{R}_{+}^{N}}|x|^{-a p}|\nabla u|^{p-2} u^{2}\left|\nabla \varphi_{\tau_{0}, s}\right|^{2} d x .
\end{aligned}
$$

Then, we get that

$$
(q-p-1) \int_{\partial \mathbb{R}_{+}^{N}} g\left(\left|x^{\prime}\right|\right)|u|^{q+1} \varphi_{\tau_{0}, s} d x^{\prime} \leq(p-1) \int_{\mathbb{R}_{+}^{N}}|x|^{-a p}|\nabla u|^{p-2} u^{2}\left|\nabla \varphi_{\tau_{0}, s}\right|^{2} d x
$$

and

$$
(r-p-1) \int_{\mathbb{R}_{+}^{N}} f(|x|)|u|^{r+1} \varphi_{\tau_{0}, s}^{2} d x \leq(p-1) \int_{\mathbb{R}_{+}^{N}}|x|^{-a p}|\nabla u|^{p-2} u^{2}\left|\nabla \varphi_{\tau_{0}, s}\right|^{2} d x .
$$

In the following, we prove that

$$
\int_{\mathbb{R}_{+}^{N}} f(|x|)|u|^{r+1} d x<\infty
$$

By (2.11), we get that

$$
\begin{aligned}
& (r-p-1) \int_{\mathbb{R}_{+}^{N}} f(|x|)|u|^{r+1} \varphi_{\tau_{0}, s}^{2} d x \\
& \quad \leq(p-1) \int_{\mathbb{R}_{+}^{N}}|x|^{-a p}|\nabla u|^{p-2} u^{2}\left|\nabla \varphi_{\tau_{0}, s}\right|^{2} d x
\end{aligned}
$$




$$
\begin{aligned}
= & (p-1) \int_{\tau_{0}<|x|<2 \tau_{0}}|x|^{-a p}|\nabla u|^{p-2} u^{2}\left|\nabla \varphi_{\tau_{0}, s}\right|^{2} d x \\
& +(p-1) \int_{s<|x|<2 s}|x|^{-a p}|\nabla u|^{p-2} u^{2}\left|\nabla \varphi_{\tau_{0}, s}\right|^{2} d x \\
\leq & (p-1)\left(\int_{\tau_{0}<|x|<2 \tau_{0}}|x|^{-a p}|\nabla u|^{p} d x\right)^{\frac{p-2}{p}}\left(\int_{\tau_{0}<|x|<2 \tau_{0}}|x|^{-a p}|u|^{p}|\nabla u|^{p} d x\right)^{\frac{2}{p}} \\
& +(p-1)\left(\int_{s \leq|x| \leq 2 s}|x|^{-a p}|\nabla u|^{p} d x\right)^{\frac{p-2}{p}}\left(\int_{s \leq|x| \leq 2 s}|x|^{-a p}|u|^{p}\left|\nabla \varphi_{r_{0, s}}\right|^{p} d x\right)^{\frac{2}{p}} \\
\leq & \left.c_{1}\left(\tau_{0}\right)^{-2 a} \int_{\tau_{0}<|x|<2 \tau_{0}}|x|^{-a p}|u|^{p}|\nabla u|^{p} d x\right)^{\frac{2}{p}}+c_{2}(s)^{-2 a-2} \int_{s \leq|x| \leq 2 s}|u|^{p} d x .
\end{aligned}
$$

By Hölder inequality, one gets

$$
\int_{s \leq|x| \leq 2 s}|u|^{p} d x \leq\left(\int_{\Omega_{2 s}} f(|x|)|u|^{r+1} d x\right)^{\frac{p}{r+1}}\left(\int_{\Omega_{2 s}} f(|x|)^{\frac{-p}{r+1-p}} d x\right)^{\frac{r+1-p}{r+1}},
$$

where $\Omega_{s}=B_{s}^{+} \backslash \overline{B_{2 \tau_{0}}^{+}}$for $s>2 \tau_{0}$, and $B_{s}^{+}$is defined in (3.1). Thus, it follows from (2.13) and (2.14) that

$$
\begin{aligned}
& \int_{\mathbb{R}_{+}^{N}} f(|x|)|u|^{r+1} d x \\
& \quad \leq c_{1}+c_{2}(s)^{-2 a-2}\left(\int_{\Omega_{2 s}} f(|x|)|u|^{r+1} d x\right)^{\frac{2}{r+1}}\left(\int_{\Omega_{2 s}} f(|x|)^{\frac{p}{r+1-p}} d x\right)^{\frac{2(r+1-p)}{p(r+1)}} .
\end{aligned}
$$

Note that $s>2 \tau_{0}>1$, then if $r>p-1+\frac{b p}{N}$, we get

$$
\int_{\Omega_{2 s}} f(|x|)^{\frac{p}{r+1-p}} d x \leq c_{3} \int_{\Omega_{2 s}}|x|^{\frac{-b p}{r+1-p}} d x \leq c_{3}|r|^{N-\frac{b p}{r+1-p}}
$$

Combining (2.15) with (2.16), we obtain

$$
\begin{aligned}
\int_{\Omega_{2 s}} f(|x|)^{\frac{p}{r+1-p}} d x & \leq c_{0}+c_{1}|2 s|^{\frac{2 N(r+1-p)-2 b p}{p(r+1)}-2(a+1)}\left(\int_{\Omega_{2 s}} f(|x|)|u|^{r+1} d x\right)^{\frac{2}{r+1}} \\
& =c_{0}+c_{1}|s|^{\theta} 2^{\theta}\left(\int_{\Omega_{2 s}} f(|x|)|u|^{r+1} d x\right)^{\frac{2}{r+1}}
\end{aligned}
$$

where

$$
\theta=\frac{2 N(r+1-p)-2 b p}{p(r+1)}-2(a+1)<0 .
$$

In the following, we will prove the first part of (2.4) by contradiction.

Suppose $\int_{\mathbb{R}_{+}^{N}} f(|x|)|u|^{r+1} d x=\infty$. Then one gets by (2.17) that there exists a constant $\alpha>0$ such that

$$
G(s)=\int_{\Omega_{s}} f(|x|)|u|^{r+1} d x \leq \alpha\left(\int_{\Omega_{2 s}} f(|x|)|u|^{r+1} d x\right)^{\frac{2}{r+1}} 2^{\theta} s^{\theta} .
$$


Integrating (2.19), we get

$$
G(s) \leq \alpha^{\gamma_{m}} 2^{\gamma_{m} \theta} s^{\gamma_{m} \theta}\left(G\left(2^{m+1} s\right)\right)^{\left(\frac{2}{r+1}\right)^{m+1}}
$$

where

$$
\beta=\frac{2}{r+1}<1, \quad \gamma_{m}=1+\beta+\beta^{2}+\cdots+\beta^{m}=\frac{1-\beta^{m}}{1-\beta} \rightarrow \frac{1}{1-\beta} \quad \text { as } m \rightarrow \infty .
$$

On the other hand, by our assumption, the solution is bounded. So, there exists $M>0$ such that $|u(x)| \leq M$ in $\partial \mathbb{R}_{+}^{\mathbb{N}}$ and

$$
\begin{aligned}
G\left(2^{m+1} s\right) & =\int_{\Omega_{2^{m+1} s}} f(|x|)|u|^{r+1} d x \leq \frac{3}{2} b_{0} M^{r+1} \int_{\Omega_{2^{m+1} s}}|x|^{b} d x \\
& =\frac{3}{2} b_{0} M^{r+1} \frac{\omega_{N}\left(2^{m+1} s\right)^{N+b}}{N+b} .
\end{aligned}
$$

Then we get from (2.20) and (2.21) that

$$
\begin{aligned}
G(s) & \leq \alpha^{\gamma_{m}} 2^{\gamma_{m} \theta} s^{\gamma_{m} \theta}\left(\frac{3}{2} b_{0} \omega_{N} \frac{\left(2^{m+1} s\right)^{N+b}}{N+b}\right)^{\left(\frac{2}{r+1}\right)^{m+1}} \\
& =c_{0} \alpha^{\gamma_{m}} 2^{\gamma_{m} \theta+(m+1)(N+b) \beta^{m+1}} s^{\gamma_{m} \theta+(N+b) \beta^{m+1}},
\end{aligned}
$$

where $\theta$ is defined as (2.18).

Note that, when $m \rightarrow \infty$,

$$
\begin{aligned}
& \gamma_{m} \theta+(m+1)(N+b) \beta^{m+1} \rightarrow \beta_{0}=\frac{p+1}{p-1} \theta, \\
& \gamma_{m} \theta+(N+b) \beta^{m+1} \rightarrow \beta_{0}=\frac{p+1}{p-1} \theta .
\end{aligned}
$$

Then, there exists $c_{2}>0$ such that

$$
G(s) \leq c_{2} s^{\frac{\beta_{0}}{2}}, \quad s>2 \tau_{0}, m>1
$$

Since $\beta_{0}<0$, (2.24) yields $G(\infty)=0$, which contradicts $\int_{\mathbb{R}_{+}^{N}} f(|x|)|u|^{r+1} d x=\infty$. Thus, we complete the proof of (i).

(ii) $1<r<p-1+\frac{b p}{N}$.

For a large $\tau_{0}>0$, we get

$$
\int_{\Omega_{2 s}} f(|x|)^{\frac{p}{r+1-p}} d x \leq c \int_{\Omega_{2 s}}|x|^{\frac{p}{r+1-p}} d x \leq c\left(2 \tau_{0}\right)^{N-\frac{b p}{r+1-p}}<1 .
$$

Then, we get from (2.15) and (2.25) that

$$
\int_{\Omega_{s}} f(|x|)|u|^{r+1} d x \leq c_{1}+c_{2}(s)^{-2 a-2}\left(\int_{\Omega_{2 s}} f(|x|)|u|^{r+1} d x\right)^{\frac{2}{r+1}}
$$


Suppose $\int_{\mathbb{R}_{+}^{N}} f(|x|)|u|^{r+1} d x=\infty$. Then there exists $\alpha>0$ such that

$$
\begin{aligned}
G(s) & =\int_{\Omega_{s}} f(|x|)|u|^{p+1} d x \leq \alpha s^{-(2 \alpha+2)}\left(\int_{\Omega_{2 s}} f(|x|)|u|^{r+1} d x\right)^{\frac{2}{r+1}} \\
& \leq \alpha^{\gamma_{m}} s^{-(2 \alpha+2) \gamma_{m}} G\left(2^{m+1} s\right)^{\beta^{m+1}}, \quad m=0,1,2, \ldots
\end{aligned}
$$

Thus, one gets from (2.21) and (2.27) that

$$
G(s) \leq c_{0} \alpha^{\gamma_{m}} s^{-(2 \alpha+2) \gamma_{m}+(N+b) \beta^{m+1}} 2^{(m+1)(N+b) \beta^{m+1}} .
$$

Since $\gamma_{m} \rightarrow \gamma_{0}=\frac{p+1}{p-1}$ as $m \rightarrow \infty$ and $\beta<1$, we obtain $G(\infty)=0$, which contradicts $\int_{\mathbb{R}_{+}^{N}} f(|x|)|u|^{r+1} d x=\infty$. The proof of (ii) is completed.

(iii) $r=p-1+\frac{b p}{N}$.

For this case, there exists a constant $c_{0}>0$ such that

$$
\int_{\Omega_{2 s}} f(|x|)^{-\frac{p}{r+1-p}} d x \leq \int_{\Omega_{2 s}}|x|^{\frac{-b p}{r+1-p}} d x \leq c_{0} \int_{2 \tau_{0}}^{2 s} \rho^{-1} d \rho \leq c_{0} s .
$$

Moreover, similar to case (i) and (ii), we can obtain

$$
G(s) \leq c_{1}+c_{2} s^{-(2 a+1)}\left(\int_{\Omega_{2 s}} f(|x|)|u|^{r+1} d x\right)^{\frac{2}{r+1}}
$$

and there exists a constant $\alpha>0$ such that

$$
\begin{aligned}
G(s) & \leq \alpha^{\gamma_{m}} s^{-(2 \alpha+1) \gamma_{m}}\left(G\left(2^{m+1} s\right)\right)^{\left(\frac{2}{r+1}\right)^{(m+1)}} \\
& \leq c_{0} \alpha^{\gamma_{m}} s^{-(2 a+1) \gamma_{m}+(N+a) \beta^{m+1}} 2^{(m+1)(N+a) \beta^{m+1}} .
\end{aligned}
$$

If $\int_{\mathbb{R}_{+}^{N}} f(|x|)|u|^{r+1} d x=\infty$, we can similarly get from $(2.31)$ that $G(\infty)=0$, which is a contraction. As a result, we complete the proof of (iii).

Next, we will prove $\int_{\partial \mathbb{R}_{+}^{N}} g\left(\left|x^{\prime}\right|\right)|u|^{q+1} d x^{\prime}<\infty$.

It follows from (2.10) that

$$
\begin{aligned}
\int_{B_{s}^{0} \backslash B_{\tau_{0}}^{0}} g\left(\left|x^{\prime}\right|\right)|u|^{q+1} d x^{\prime} & \leq \int_{\partial \mathbb{R}_{+}^{N}} g\left(\left|x^{\prime}\right|\right)|u|^{q+1} \phi^{2} d x^{\prime} \leq \int_{\mathbb{R}_{+}^{N}}|x|^{-a p}|\nabla u|^{p-2} u^{2}|\nabla \phi|^{2} d x \\
& \leq \frac{2}{s^{2}}\left(\int_{\Omega_{2 s}}|x|^{-a p}|\nabla u|^{p} d x\right)^{\frac{p-2}{p}}\left(\int_{\Omega_{2 s}}|x|^{-a p}|u|^{p} d x\right)^{\frac{2}{p}} \\
& \leq c s^{-2-2 a}\left(\int_{\Omega_{2 s}} f(|x|)|u|^{r+1} d x\right)^{\frac{2}{r+1}}\left(\int_{\Omega_{2 s}} f(|x|)^{\frac{-p}{r+1-p}} d x\right)^{\frac{2(r+1-p)}{p(r+1)}} \\
& \leq c s^{\theta} .
\end{aligned}
$$

Noting that $\theta<0$, we get from (2.32) that

$$
\int_{\mathbb{R}^{N-1} \backslash B_{\tau_{0}}} g\left(\left|x^{\prime}\right|\right)|u|^{q+1} d x^{\prime}=0 .
$$

Furthermore, we get the second claim in (2.4), and the proof of this lemma is completed 


\section{The proof of Theorem 1}

In this part, we will complete the proof of Theorem 1 . To make the proof clear, we give the following symbols;

$$
\begin{aligned}
& S_{s}^{+}=\left\{x=\left(x^{\prime}, x_{N}\right) \in \mathbb{R}^{N-1} \times \mathbb{R}^{+}:|x|=s\right\}, \\
& B_{s}^{0}=\left\{x=\left(x^{\prime}, 0\right), x^{\prime} \in \mathbb{R}^{N-1}:\left|x^{\prime}\right|<s\right\}, \\
& \partial B_{s}^{0}=\left\{x=\left(x^{\prime}, 0\right), x^{\prime} \in \mathbb{R}^{N-1}:\left|x^{\prime}\right|=s\right\}, \\
& B_{s}^{+}=\left\{x=\left(x^{\prime}, x_{N}\right) \in \mathbb{R}^{N-1} \times \mathbb{R}^{+}:|x|<s\right\} .
\end{aligned}
$$

It is obvious that $\partial B_{R}^{+}=S_{R}^{+} \cup B_{R}^{0}$, where $R \in \mathbb{R}^{+}$. In order to prove the nonexistence of solutions, we need to establish the following Pohozaev identity for problem (1.1).

Lemma 3.1 Let $u$ be a solution of (1.1), then for any $R>0$ the following equality holds:

$$
\begin{aligned}
\left(\frac{N}{p}\right. & -1-a) \int_{B_{R}^{+}}|x|^{-a p}|\nabla u|^{p} d x-\frac{1}{r+1} \int_{B_{R}^{+}}\left[N f(|x|)+|x| f^{\prime}(|x|)\right]|u|^{r+1} d x \\
& -\frac{1}{q+1} \int_{B_{R}^{0}}|u|^{q+1}\left[(N-1) g\left(\left|x^{\prime}\right|\right)+\left|x^{\prime}\right| g^{\prime}(|x|)\right] d x^{\prime}-\frac{R}{p} \int_{B_{R}^{0}}|x|^{-a p}|\nabla u|^{p} d x \\
= & \frac{-R}{r+1} \int_{\partial B_{R}^{+}} f(|x|)|u|^{r+1} d S+\frac{R}{p} \int_{S_{R}^{+}}|x|^{-a p}|\nabla u|^{p} d S \\
& -\frac{R}{q+1} \int_{\partial B_{R}^{0}} g(|x|)|u|^{q+1} d \sigma .
\end{aligned}
$$

Proof Multiplying (1.1) by $x \cdot \nabla u$ and integrating, we get

$$
-\sum_{j=1}^{N} \int_{B_{R}^{+}} \frac{\partial}{\partial x_{j}}\left(|x|^{-a p}|\nabla u|^{p-2} \frac{\partial u}{\partial x_{j}}\right) x \cdot \nabla u d x=\sum_{i=1}^{N} \int_{B_{R}^{+}} f(|x|)|u|^{r-1} u x_{i} \frac{\partial u}{\partial x_{i}} d x .
$$

For the right-hand side of (3.3), we have

$$
\begin{aligned}
\sum_{i=1}^{N} \int_{B_{R}^{+}} f(|x|)|u|^{r-1} u x_{i} \frac{\partial u}{\partial x_{i}} d x \\
=\frac{1}{r+1} \sum_{i=1}^{N} \int_{B_{R}^{+}} \frac{\partial}{\partial x_{i}}\left(x_{i} f(|x|)|u|^{r+1}\right) d x \\
\quad-\frac{N}{r+1} \int_{B_{R}^{+}} f(|x|)|u|^{r+1} d x-\sum_{i=1}^{N} \int_{B_{R}^{+}}\left(\int_{0}^{u} x_{i} f^{\prime}(|x|) \frac{x_{i}}{|x|}|s|^{r-1} s d s\right) d x \\
=\frac{1}{r+1} \sum_{i=1}^{N} \int_{\partial B_{R}^{+}} x_{i} \cdot v_{i} f(|x|)|u|^{r+1} d S-\frac{N}{r+1} \int_{B_{R}^{+}} f(|x|)|u|^{r+1} d x \\
\quad-\frac{1}{r+1} \int_{B_{R}^{+}} f^{\prime}(|x|)|x||u|^{r+1} d x \\
=\frac{R}{r+1} \int_{\partial B_{R}^{+}} f(|x|)|u|^{r+1} d S \\
\quad-\frac{1}{r+1} \int_{B_{R}^{+}}\left(N f(|x|)+|x| f^{\prime}(|x|)|u|^{r+1}\right) d x .
\end{aligned}
$$


For the left part of (3.3), we have

$$
\begin{aligned}
- & \sum_{j=1}^{N} \int_{B_{R}^{+}} \frac{\partial}{\partial x_{j}}\left(|x|^{-a p}|\nabla u|^{p-2} \frac{\partial u}{\partial x_{j}}\right) x \cdot \nabla u d x \\
= & -\sum_{j=1}^{N} \int_{B_{R}^{+}} \frac{\partial}{\partial x_{j}}\left(|x|^{-a p}|\nabla u|^{p-2} \frac{\partial u}{\partial x_{j}} x \cdot \nabla u\right) d x \\
& +\int_{B_{R}^{+}}|x|^{-a p}|\nabla u|^{p-2} \frac{\partial u}{\partial x_{j}} \sum_{i=1}^{N}\left(\delta_{i j} \frac{\partial u}{\partial x_{i}}+x_{i} \frac{\partial^{2} u}{\partial x_{i} \partial x_{j}}\right) d x \\
= & -\sum_{j=1}^{N} \int_{\partial B_{R}^{+}}\left(|x|^{-a p}|\nabla u|^{p-2} \frac{\partial u}{\partial x_{j}} v_{j} x \cdot \nabla u\right) d S+\int_{B_{R}^{+}}|x|^{-a p}|\nabla u|^{p} d x \\
& +\sum_{j=1}^{N} \int_{B_{R}^{+}}|x|^{-a p}|\nabla u|^{p-2} \frac{\partial u}{\partial x_{j}} \sum_{i=1}^{N} x_{i} \frac{\partial^{2} u}{\partial x_{i} \partial x_{j}} d x .
\end{aligned}
$$

For the first and third terms of the right-hand side of (3.5), we get

$$
\begin{aligned}
& \sum_{j=1}^{N} \int_{\partial B_{R}^{+}}\left(|x|^{-a p}|\nabla u|^{p-2} \frac{\partial u}{\partial x_{j}} v_{j} x \cdot \nabla u\right) d S \\
& =\int_{S_{R}^{+}}|x|^{-a p}|\nabla u|^{p} d S \\
& +\sum_{i=1}^{N-1} \int_{B_{R}^{0}} g\left(\left|x^{\prime}\right|\right)|u|^{q-1} u x_{i} \frac{\partial u}{\partial x_{i}} d x^{\prime} \\
& =\int_{S_{R}^{+}}|x|^{-a p}|\nabla u|^{p} d S+\frac{1}{q+1} \sum_{i=1}^{N-1} \int_{B_{R}^{0}}\left[\frac{\partial}{\partial x_{i}}\left(x_{i} g\left(\left|x^{\prime}\right|\right)|u|^{q+1}\right)-|u|^{q+1} \frac{\partial}{\partial x_{i}}\left(x_{i} g\left(\left|x^{\prime}\right|\right)\right] d x^{\prime}\right. \\
& =\int_{S_{R}^{+}}|x|^{-a p}|\nabla u|^{p} d S+\frac{1}{q+1} \sum_{i=1}^{N-1} \int_{\partial B_{R}^{0}} x_{i} g(|x|)|u|^{q+1} v_{i} d \sigma \\
& -\frac{1}{q+1} \sum_{i=1}^{N-1} \int_{B_{R}^{0}}|u|^{q+1} \frac{\partial}{\partial x_{i}}\left(x_{i} g\left(\left|x^{\prime}\right|\right)\right) d x^{\prime} \\
& =\int_{S_{R}^{+}}|x|^{-a p}|\nabla u|^{p} d S+\frac{1}{q+1} \int_{\partial B_{R}^{0}}\left(x \cdot v g(|x|)|u|^{q+1}\right) d \sigma \\
& -\frac{1}{q+1} \sum_{i=1}^{N-1} \int_{B_{R}^{0}}|u|^{q+1}\left[g\left(\left|x^{\prime}\right|\right)+x_{i} g^{\prime}\left(\left|x^{\prime}\right|\right) \frac{x_{i}}{|x|}\right] d x^{\prime} \\
& =\int_{S_{R}^{+}}|x|^{-a p}|\nabla u|^{p} d S++\frac{R}{q+1} \int_{\partial B_{R}^{0}} g(|x|)|u|^{q+1} d \sigma \\
& -\frac{1}{q+1} \int_{B_{R}^{0}}\left[(N-1) g\left(\left|x^{\prime}\right|\right)+\left|x^{\prime}\right| g^{\prime}\left(\left|x^{\prime}\right|\right)\right] d x^{\prime}
\end{aligned}
$$

and

$$
\sum_{j=1}^{N} \int_{B_{R}^{+}}|x|^{-a p}|\nabla u|^{p-2} \frac{\partial u}{\partial x_{j}} \sum_{i=1}^{N} x_{i} \frac{\partial^{2} u}{\partial x_{i} \partial x_{j}} d x
$$




$$
\begin{aligned}
= & \frac{1}{p} \sum_{i=1}^{N} \int_{B_{R}^{+}}|x|^{-a p} x_{i} \frac{\partial}{\partial x_{i}}\left(|\nabla u|^{p}\right) d x \\
= & \frac{-N}{p} \int_{B_{R}^{+}}|x|^{-a p}|\nabla u|^{p} d x+\frac{1}{p} \sum_{i=1}^{N} \int_{\partial B_{R}^{+}}|x|^{-a p}|\nabla u|^{p} x_{i} \vartheta_{i} d S \\
& +a \sum_{i=1}^{N} \int_{B_{R}^{+}} x_{i}|x|^{-a p-1} \frac{x_{i}}{|x|}|\nabla u|^{p} d x \\
= & \frac{-N}{p} \int_{B_{R}^{+}}|x|^{-a p}|\nabla u|^{p} d x+\frac{R}{p} \int_{\partial B_{R}^{+}}|x|^{-a p}|\nabla u|^{p} d S+a \int_{B_{R}^{+}}|x|^{-a p}|\nabla u|^{p} d x .
\end{aligned}
$$

Thus, (3.2) follows from (3.3)-(3.7).

Now, we give the proof of Theorem 1.

Multiplying (1.1) by $u$ and integrating, one gets that

$$
\int_{\mathbb{R}_{+}^{N}}|x|^{-a p}|\nabla u|^{p} d x=\int_{\mathbb{R}_{+}^{N}} f(|x|)|u|^{r+1} d x+\int_{\partial \mathbb{R}_{+}^{N}} g\left(\left|x^{\prime}\right|\right)|u|^{q+1} d x^{\prime} .
$$

We need to prove

$$
\liminf _{R \rightarrow \infty} R\left(\int_{\partial B_{R}^{+}} f(|x|)|u|^{r+1} d S+\int_{\partial B_{R}^{+}}|x|^{-a p}|\nabla u|^{p} d S+\int_{\partial B_{R}^{0}} g(|x|)|u|^{q+1} d \sigma\right)=0 .
$$

Assume on the contrary that (3.9) is wrong. Then there exists a constant $\delta>0$ such that

$$
\liminf _{R \rightarrow \infty} R\left(\int_{\partial B_{R}^{+}} f(|x|)|u|^{r+1} d S+\int_{\partial B_{R}^{+}}|x|^{-a p}|\nabla u|^{p} d S+\int_{\partial B_{R}^{0}} g(|x|)|u|^{q+1} d \sigma\right)=\delta .
$$

Then, there exists $R_{0} \in \mathbb{R}^{+}$such that

$$
R\left(\int_{\partial B_{R}^{+}} f(|x|)|u|^{r+1} d S+\int_{\partial B_{R}^{+}}|x|^{-a p}|\nabla u|^{p} d S+\int_{\partial B_{R}^{0}} g(|x|)|u|^{q+1} d \sigma\right)>\delta / 2
$$

for all $R>R_{0}$.

Writing $R_{n}=R_{0}+n, n=1,2, \ldots$, there exists $\zeta_{n} \in\left(R_{n-1}, R_{n}\right)$ such that for $n=1,2, \ldots$, there holds

$$
\begin{aligned}
& \int_{R_{n-1}}^{R_{n}}\left(\int_{\partial B_{R}^{+}} f(|x|)|u|^{r+1} d S+\int_{\partial B_{R}^{+}}|x|^{-a p}|\nabla u|^{p} d S+\int_{\partial B_{R}^{0}} g(|x|)|u|^{q+1} d \sigma\right) d R \\
& \quad=\zeta_{n}\left(\int_{\partial B_{R}^{+}} f(|x|)|u|^{r+1} d S+\int_{\partial B_{R}^{+}}|x|^{-a p}|\nabla u|^{p} d S+\int_{\partial B_{R}^{0}} g(|x|)|u|^{q+1} d \sigma\right) \\
& \quad>\delta / 2 .
\end{aligned}
$$

Furthermore, we get

$$
\int_{0}^{\infty}\left(\int_{\partial B_{R}^{+}} f(|x|)|u|^{r+1} d S+\int_{\partial B_{R}^{+}}|x|^{-a p}|\nabla u|^{p} d S+\int_{\partial B_{R}^{0}} g(|x|)|u|^{q+1} d \sigma\right) d R
$$




$$
\begin{aligned}
& \geq \sum_{n=2}^{\infty} \int_{R_{n-1}}^{R_{n}}\left(\int_{\partial B_{R}^{+}} f(|x|)|u|^{r+1} d S+\int_{\partial B_{R}^{+}}|x|^{-a p}|\nabla u|^{p} d S+\int_{\partial B_{R}^{0}} g(|x|)|u|^{q+1} d \sigma\right) d R \\
& =\infty
\end{aligned}
$$

which contracts the result of Lemma 2.2, and we get (3.9).

Thus, letting $R \rightarrow \infty$ in (3.2), it follows that

$$
\begin{aligned}
& \left(\frac{N}{p}-1-a\right) \int_{\mathbb{R}_{+}^{N}}|x|^{-a p}|\nabla u|^{p} d x-\frac{1}{r+1} \int_{\mathbb{R}_{+}^{N}}|u|^{r+1}\left[N f(|x|)+|x| f^{\prime}(|x|)\right] d x \\
& \quad=\frac{1}{q+1} \int_{\partial \mathbb{R}_{+}^{N}}|u|^{q+1}\left[(N-1) g\left(\left|x^{\prime}\right|\right)+\left|x^{\prime}\right| g^{\prime}\left(\left|x^{\prime}\right|\right)\right] d x^{\prime} .
\end{aligned}
$$

For any $\eta \in \mathbb{R}$, we get from (3.8) and (3.14) that

$$
\begin{aligned}
\left(\frac{N}{p}\right. & -1-a-\eta) \int_{\mathbb{R}_{+}^{N}}|x|^{-a p}|\nabla u|^{p} d x-\left(\frac{N}{r+1}-\eta\right) \int_{\mathbb{R}_{+}^{N}}|u|^{r+1} f(|x|) d x \\
& -\int_{\mathbb{R}_{+}^{N}}|u|^{r+1}|x| f^{\prime}(|x|) d x \\
= & \left(\frac{N-1}{q+1}-\eta\right) \int_{\partial \mathbb{R}_{+}^{N}}|u|^{q+1} g\left(\left|x^{\prime}\right|\right) d x^{\prime}+\frac{1}{q+1} \int_{\partial \mathbb{R}_{+}^{N}}\left|x^{\prime}\right| g^{\prime}\left(\left|x^{\prime}\right|\right) d x^{\prime} .
\end{aligned}
$$

Particularly, when $\eta=\frac{N}{p}-1-a$, it follows from $\left(A_{3}\right)$ that $u \equiv 0$ in $\mathbb{R}_{+}^{N}$. Thus, we complete the proof.

\section{Acknowledgements}

The authors would like to express their sincere gratitude to the anonymous reviewer for the valuable comments and suggestions. The authors thanks for the support of the funding.

\section{Funding}

This paper is supported by the National Natural Science Foundation of China (Grant No. 11461016); The Natural Science Foundation of Shandong Province (Grant No. ZR2016AQ04); The Advanced Talents Foundation of QAU (Grant No. 6631115047).

\section{Availability of data and materials}

Data sharing is not applicable to this article as no datasets were generated or analyzed during the current study.

\section{Competing interests}

The authors declare that they have no competing interests.

\section{Authors' contributions}

Each author contributed equally to each part of this study, all authors read and approved the final manuscript.

\section{Author details}

${ }^{1}$ Science and Information College, Qingdao Agricultural University, Qingdao, P.R. China. ${ }^{2}$ Shan Dong University of Science and Technology, Qingdao, P.R. China.

\section{Publisher's Note}

Springer Nature remains neutral with regard to jurisdictional claims in published maps and institutional affiliations.

Received: 7 January 2019 Accepted: 14 March 2019 Published online: 19 March 2019

\section{References}

1. Liouville, J.: Remarques de M. Liouville sur "Construction géométrique des amplitudes dans les fonctions elliptiques" par M. Charles. C. R. Acad. Sci. Paris 19, 1261-1263 (1844)

2. Bahri, A., Lions, P.L.: Solutions of superlinear elliptic equations and their Morse indices. Commun. Pure Appl. Math. 45, 1205-1215 (1992)

3. Chen, W., Li, C.: Classification of solutions of some nonlinear elliptic equations. Duke Math. J. 63, 615-622 (1991) 
4. Damascelli, L., Gladiali, F.: Some nonexistence results for positive solutions of elliptic equations in unbounded domains. Rev. Mat. Iberoam. 20,67-86 (2004)

5. Li, Y., Zhang, L.: Liouville-type theorems and Harnack-type inequalities for semilinear elliptic equations. J. Anal. Math 90, 27-87 (2003)

6. Dancer, E.N.: Some notes on the method of moving planes. Bull. Aust. Math. Soc. 46, 425-434 (1992)

7. Yu, X.: Liouville type theorem for nonlinear elliptic equation with general nonlinearity. Discrete Contin. Dyn. Syst., Ser. A 34, 4947-4966 (2014)

8. Yu, X: Liouville theorem for elliptic equations with nonlinear boundary value conditions and finite Morse indices J. Math. Anal. Appl. 421, 436-443 (2015)

9. Gidas, B., Spruck, J.: Global and local behavior of positive solutions of nonlinear elliptic equations. Commun. Pure Appl. Math. 24, 525-598 (1981)

10. Phan, Q.H., Souplet, P.H.: Liouville-type theorems and bounds of solutions of Hardy-Hénon equations. J. Differ. Equ. 252, 2544-2562 (2012)

11. Dancer, E.N., Du, Y.H., Guo, Z.M.: Finite Morse index solutions of an elliptic equation with supercritical exponent. J. Differ. Equ. 250, 3281-3310 (2011)

12. Bidaut-Veron, M.F., Giacomini, H.: A new dynamical approach of Emden-Fowler equations and systems. Adv. Differ. Equ. 15, 1033-1082 (2010)

13. D'Ambrosio, L., Mitidieri, E.: Quasilinear elliptic equations with critical potentials. Adv. Nonlinear Anal. 6, 147-164 (2017)

14. D'Ambrosio, L., Mitidieri, E.: Uniqueness and comparison principles for semilinear equations and inequalities in Carnot groups. Adv. Nonlinear Anal. 7, 313-325 (2018)

15. Birindelli, I., Galise, G., Leoni, F.: Liouville theorems for a family of very degenerate elliptic nonlinear operators. Nonlinear Anal. 161, 198-211 (2017)

16. Chen, C.S., Song, H.X., Yang, H.W.: Liouville-type theorems for stable solutions of singular quasilinear elliptic equations in $\mathbb{R}^{N}$. Electron. J. Differ. Equ. 2018, 81 (2018)

17. Serrin, J., Zou, H.: The existence of positive entire solutions of elliptic Hamiltonian system. Commun. Partial Differ. Equ. 23, 375-398 (1998)

18. Souplet, P.: The proof of the Lane-Emden conjecture in four space dimensions. Adv. Math. 221, 1409-1437 (2009)

19. Zhang, L.Z., Yu, M., He, J.M.: A Liouville theorem for a class of fractional systems in $\mathbb{R}_{+}^{N}$. J. Differ. Equ. 263, 6025-6065 (2017)

20. Duong, A.T., Phan, Q.H.: Liouville type theorem for nonlinear elliptic system involving Grushin operator. J. Math. Anal. Appl. 454, 785-801 (2017)

21. Hu, L.G.: Liouville-type theorems for the fourth order nonlinear elliptic equation. J. Differ. Equ. 256, 1817-1846 (2014)

22. Dávila, J., Dupaigne, L., Wang, K.L., Wei, J.C.: A monotonicity formula and a Liouville-type theorem for a fourth order supercritical problem. Adv. Math. 258, 240-285 (2014)

23. Farina, A.: On the classification of solutions of the Lane-Emden equation on unbounded domains of $\mathbb{R}^{N}$. J. Math. Pures Appl. 87, 537-561 (2007)

24. Chen, C.S., Song, H.X., Yang, H.W.: Liouville type theorems for stable solutions of $p$-Laplace equation in $\mathbb{R}^{N}$. Nonlinear Anal. 160, 44-52 (2017)

\section{Submit your manuscript to a SpringerOpen ${ }^{\circ}$ journal and benefit from:}

- Convenient online submission

- Rigorous peer review

- Open access: articles freely available online

- High visibility within the field

- Retaining the copyright to your article

Submit your next manuscript at $\gg$ springeropen.com 